\title{
Failure of Semi-infinite Beams of Variable Thickness and Curvature on Elastic Foundation under Contact Loading
}

\author{
João Batista De Aguiar ${ }^{1}$ and José Manoel De Aguiar ${ }^{2}$ \\ 1. Department of Engenharia AeroEspacial,Universidade Federal do ABC, Santo André 09210-170, Brazil \\ 2. Department of Ensino Geral, Faculdade de Tecnología de São Paulo, São Paulo 01124-060, Brazil
}

\begin{abstract}
In early winter it is usual, in cold regions, that ice features approach offshore structures, like offshore platforms, impacting them, in a slow process of constant deformation build up. Interaction follows, in many cases, up to the point where ice-failure caused by bending fracture takes place. This supposes very large contact forces that the structure has to resist. Therefore, quantification of these efforts is of vital importance to the structural design of platforms. In several designs, these platforms are constructed with inclined walls so as to cause ice to fail in a flex-compression mode. In such a case the ice feature is analyzed as a beam constituted of a linear elastic material in brittle state with constant ice thickness. The simplification renders the problem solvable in a close form. However, this hypothesis goes against field observations. Marine currents action, wind and the sequence of contacts among features lead to thickness variations. Here this factor is addressed in the construction of a model, for harmonic forms of variation of thickness profile, and the accompanying curvature variations, whose solution determines field variables used to address the failure question. Due to the deformation dependency of the loading, a numerical scheme for the two-point boundary value problem in the semi-infinite space is developed. Failure pressures are computed based on a Rankine locus of failure. Variations of the order of $20 \%$ in the failure loads, as compared to the uniform beam model, are observed.
\end{abstract}

Key words: Ice beams, thickness variation, elastic behavior, frictional contact, bending, failure loads.

\section{Introduction}

Offshore facilities in ice infested waters are constructed with diverse geometric forms. Transversely sectional forms range from rectangular to circular. Vertically variations go from cylindrical to conical among others. No matter the form, however, inclined walls are included in many designs in an effort to induce ice failure in a compressive-flexural manner, thus reducing stresses in the structure. Failure occurs as ice sheets, with irregular geometry, ride the walls up dissipating energy in friction, deforming in the way, until field stresses reach values, at critical points, that lead to fracture. It is a continuous process, difficult to model given the large number of variables to deal with.

Usually, this sort of problem is modeled using different approaches as a wide beam or plate, contact

\footnotetext{
Corresponding author: João Batista De Aguiar, Ph.D., research field: applied mechanics. E-mail: joao.aguiar@ufabc.edu.br.
}

loading aligned with, or inclined with respect to, the boarder wall direction, thus leading to a 2D or 3D beam model. Timewise, problem may be considered as a static or dynamic one, depending upon the severity of impact. Geometry of what may be modeled as a beam occurs in nature under diverse forms. Width is never constant, and thickness specially presents variations [1]. It is therefore not realistic to model contact problems with offshore platforms assuming constancy of thickness, with no initial curvature present, even though such a hypothesis unveils a close form solution to the problem-uniform geometry beams analyzed as a linear elastic material in brittle state have a known solution [2]. Furthermore, this restriction goes against field observations, marine currents action, wind and the sequence of contacts among features lead to thickness variations.

In this work geometry effect is addressed and the construction of a mechanical model, for harmonic 

on Elastic Foundation under Contact Loading

forms of variation of the thickness profile, with the accompanying curvature variations produced. Solution of determines field variables such as displacements and internal resultants that make it possible verify fail safe-unsafe states in a locus of failure. Implementation of the constructed model requires an analytical-numerical solution because loading terms are deformation dependent. Use of the usual finite element approach, due to the semi-infinite characterization of the problem, makes it complicated mostly because of the profile variations. For these reasons, the two-point boundary value is solved in an iterative manner, developed in detail ahead. Coded in FORTRAN, the procedure entails fast convergence and easy of use.

Results provided by analysis are classified in two categories: in one only effects of thickness variations are present; in the other initial curvature of ice is also present. They have comparable importance, causing together up to $20 \%$ variations in the values of failure loads, when compared to the uniform beam, the one with constant thickness and no initial curvature.

\section{Formulation}

\subsection{Boundary Value Problem}

Upon contact with the inclined wall of an offshore structure, loading of ice beam, driven by wind and currents starts. Equilibrium of every sectional element of it, supposed constant the width $b_{0}$ but variable the height $h, h=h(y)$, with flotation modeled by means of a linear elastic foundation of constant $k$ (Fig. 1), is given by Ref. [3]:

$$
\partial_{, y y}\left[\left(\frac{t}{t_{0}}\right)^{3}\left(\kappa-\kappa_{i}\right)\right]+4 \gamma_{0}{ }^{4}\left(\kappa-\kappa_{i}\right)+4 \delta_{0}{ }^{4}\left(w-w_{i}\right)=0
$$

where, $k=\partial$,yy $(w)$ is the curvature and $k_{i}=\partial$,yy $\left(w_{i}\right)$ is the initial curvature. Coefficients are as following:

$$
\gamma_{0}=\left(\frac{n_{0}}{4 E^{\prime} i_{0}}\right)^{\frac{1}{4}} ; n_{0} \geqq 0, \quad \delta_{0}=\left(\frac{k}{4 E^{\prime} i_{0}}\right)^{\frac{1}{4}}
$$

where, $n_{0}$ is the value of the normal force per unit width at the origin and $\bar{h}$ is the mean thickness, measured by means of the thickness profile function $t$, so that $h=\bar{h} t$. The bending stiffness per unit width $i_{0}$ and $E^{\prime}$, the equivalent Young's modulus of the beam, complete the set, hence,

$$
n_{0}=\frac{N_{0}}{b_{0}} ; i_{0}=\frac{h_{0}{ }^{3}}{12}=\frac{\bar{h}^{3} t_{0}{ }^{3}}{12} ; E^{\prime}=\frac{E}{\left(1-v^{2}\right)}
$$

where, $E$ is the elastic modulus of the material, $v$ is Poisson's ratio, introduced to take into account the transversal curvature effect along wide beams and $t_{0}$ is the thickness at origin. If in particular the beam profile is symmetric, then the initial curvatures $k_{1}$ caused by thickness variations are null, with no curvature-thickness coupling present (Fig. 2).

Interface loading due to impact of the beam with the inclined wall has a complex stress distribution, dependent upon the profile of the beam there. It can, nonetheless, be expressed in a per unit width fashion by a set of stress resultants including a shear force $v_{0}$ and a bending moment $m_{0}$, both related to the normal resultant $n_{0}$ :

$$
\begin{gathered}
y=0 ; m_{0}=-E^{\prime} i_{0} \partial_{, y y}\left(w_{r}\right)_{0} ; \quad w_{r}=w-w_{i} \\
v_{0}=-E^{\prime} i_{0} \partial_{, y y y}\left(w_{r}\right)_{0}-\frac{3}{t} \partial_{, y}(t)_{0} m_{0}-n_{0} \partial_{, y}\left(w_{r}\right)_{0}
\end{gathered}
$$

Function $w_{i}$ represents the initial curvature of the equal area axis, corresponding to line of mean thickness $\bar{h}$. Lateral displacements are denoted by $w$. Both are measured with respect to the water line WL. These equations show that loading relies on the free parameter $n_{0}$, related to the solution of the in-plane problem. They also reveal dependence upon rotation, curvature and rate of curvature at the origin.

At the other end, the far end, for the semi-infinite model $L \rightarrow \infty$, and regularity conditions apply:

$$
\begin{gathered}
y=L ; \quad w_{L}=0 \\
\partial_{, y}(w)_{L}=0
\end{gathered}
$$

\subsection{System Transformation}

Solution of Eq. (1), subjected to the stated boundary conditions, Eqs. (4) and (5) may be accomplished by a transformation of this equation into a set of four equations. For that sake, let $u$ be the vector, 




Fig. 1 Beam element with thickness variation $\left\langle b_{0}, h(y), L>\right.$, under contact loading with offshore structure $\left\langle n_{0}, v_{0}, m_{0}\right\rangle$.

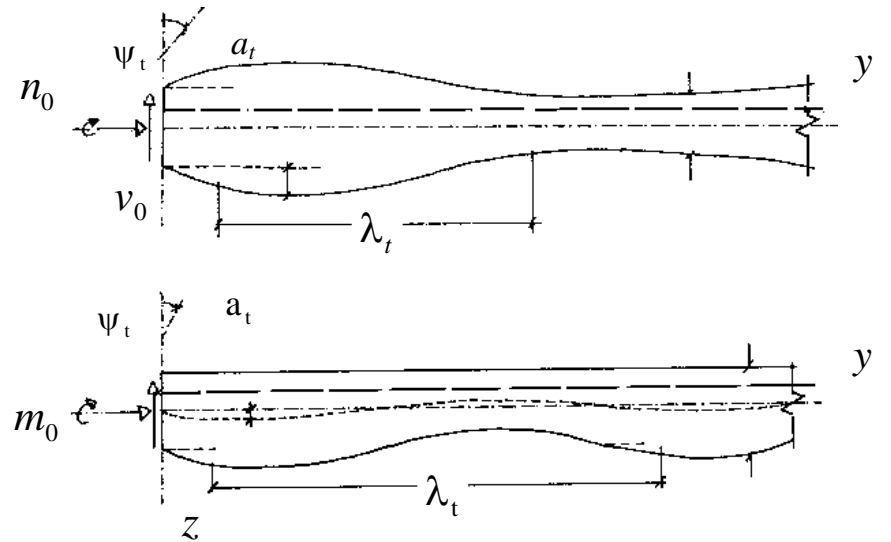

Fig. 2 Profiles of variable thickness beam $\left\langle a_{t}, \lambda_{t}, \psi_{t}>\right.$ : symmetric case, $w_{i}=0$, and anti-symmetric case, $w_{i} \neq 0$.

$$
\{u\}=\left[\partial_{, y y y}(w) \cdot \partial_{, y y}(w) \cdot \partial_{, y}(w) \cdot w\right] ; w=\hat{w}_{t}(y ; b t ; t)
$$

here represented in row form, and dependent upon positions $y$, boundary terms b.t. and profile $t$. With the above definition Eq. (1) will take the form [4]:

$\left\{u^{\prime}\right\}=[K]\{u\}+\left\{u_{i}\right\} ; u_{n}^{\prime}=\partial_{, y}\left(u_{n}\right) ; n=1,2,3,4$

Interface rotation $\theta_{0}=\partial_{, y}(w)_{0}$, result of local deformation, plus regularity conditions, will cast boundary conditions into the form:

$$
\{\varphi\}=[A]\left\{u_{0}\right\}+[B]\left\{u_{L}\right\}+\{\alpha\}-\{\beta\} ;\{\varphi\}=\{0\}
$$

where, matrices $[A]$ and $[B]$ are related to geometry and loading whereas vectors $\{\alpha\}$ and $\{\beta\}$ are dependent upon initial curvature of the beam.

\subsection{Numerical Solution}

Solution of the above system depends, for every value of $n_{0}$, on the determination of the exact vector $\{u\}$ that makes $\{\varphi\}$ null. Therefore, it requires specification of the left and right end vectors, whose values are not known in advance. So as a solution procedure, a numerical trial and error type of approach may be applied, using a Newton scheme of solution [5]. Hence if at some iteration $j$,

$$
\begin{gathered}
\left\{\varphi^{j}\right\} \neq\{0\} ;\left\{\varphi^{j}\right\}=[A]\left\{u_{0}{ }^{j}\right\}+[A]\left\{u_{n}{ }^{j}\right\}+\left\{\alpha^{j}\right\}-\left\{\beta^{j}\right\} ; \\
j=1,2, \ldots, M
\end{gathered}
$$

over a discretized space containing $N+1$ stations, $\left\{y_{i}\right\}$, $i=0,1,2, \ldots, N$, being

$$
\begin{gathered}
\left\{u^{j}{ }_{0}\right\}=\left\{u^{j}\left(y_{0}\right)\right\} ;\left\{u^{j}{ }_{N}\right\}=\left\{u^{j}\left(y_{N}\right)\right\} \\
y_{0}=0 ; y_{n}=L ; \forall j
\end{gathered}
$$

Then requiring that boundary conditions be satisfied in the next iteration means that:

$\left\{\varphi^{j+1}\right\}=\{0\} ;$

$\left\{\varphi^{j+1}\right\}=[A]\left\{u_{0}^{j+1}\right\}+[B]\left\{u_{N}^{j+1}\right\}+\left\{\alpha^{j+1}\right\}-\left\{\beta^{j+1}\right\}$

What asks for, using Taylor expansion, an increment 
$\Delta\left\{u_{0}^{j+1}\right\}$

$$
\Delta\left\{u_{o}{ }^{j+1}\right\}=-\left[\partial_{\left\{u_{0}{ }^{j+1}\right\}}\left(\varphi_{N}^{j+1}\right)\right]^{-1} \cdot\left\{\varphi_{N}^{j}\right\}
$$

where, the partial derivative with respect to the trial vector is

$$
\begin{gathered}
{\left[\partial_{\left\{u_{0}^{j+1}\right\}}\left\{\varphi^{j+1}\right\}\right]=[A][I]+[B]\left[\partial_{\left\{u_{0}^{j+1}\right\}}\left\{u_{N}^{j+1}\right\}\right]} \\
+\left[\partial_{\left\{u_{0}^{j+1}\right\}}\left\{\alpha^{j+1}\right\}\right]-\left[\partial_{\left\{u_{0}^{j+1}\right\}}\left\{\beta^{j+1}\right\}\right]
\end{gathered}
$$

In this expression the identity matrix $[I]$ appears first. In the middle term the derivative at the far end with respect to the first one gives rise to a transfer matrix: $\partial_{\left\{u_{0}^{j+1}\right\}}\left\{u_{N}^{j+1}\right\}=T_{0}^{N}$.

\section{Model Parameters}

\subsection{Loading Specification}

Interface loading resultants depend on the coefficient of friction $\mu$ between ice and the rigid wall of the platform, its slope angle $\phi$ and the coefficient of eccentricity $\zeta$ as shown in Fig. 3. Solving the normal $r_{0} e_{n}$ and tangential $\mu r_{0} e_{\mathrm{t}}$ to the inclined wall in terms of its horizontal and vertical components, while denoting the rotation at the origin by $\theta_{0}$, leads to:

$$
\begin{aligned}
& n_{0}=-z_{0} \sin \theta_{0}+y_{0} \cos \theta_{0} ; \\
& v_{0}=z_{0} \cos \theta_{0}+y_{0} \sin \theta_{0} ; m_{0}=-n_{0} e
\end{aligned}
$$

being $e=\zeta h_{0} ;-0.5 \leq \zeta \leq 0.5$ the eccentricity. It depends on the form of the beam at the origin section. Sticking-slipping conditions require that:

$$
\begin{gathered}
v_{0} \leq n_{0} \tan \left(\phi_{t}\right) \text { when } \phi_{t} \leq \rho ; \\
v_{0} \leq n_{0} \tan \left(\phi_{t}-\rho\right) \text { when } \phi_{t} \geq \rho
\end{gathered}
$$

where, $\rho$ is a material parameter related to the way ice and wall interact, surface roughness and temperature among other factors. It is important to notice that $\phi_{t}=\theta_{0}+\phi$. Table 1 presents the values considered in the present analysis, where beam aspect ratios $h_{0} / b_{0}=\{1,2,4\}$ were employed.

\subsection{Material Parameters}

Ice is a quite complex material, whose constitutive equation depends on the type of microstructure considered, time of the year, form of response sought, etc.. For ice features in salt water, buoyancy associates to the foundation constant $k$ the value $\gamma_{s w}=1.0045$ e $+4 \mathrm{~Pa} / \mathrm{m}$. For ice in brittle state, Table 2 presents some average values of the properties needed to the model [6]. These properties also depend on temperature and rate of loading, not considered in the study.

\subsection{Contact Variables}

In the field, the monitored variable $r_{0}$, the intensity of contact, has to be used to compute the interface normal $n_{0}$. However, because the displacements do depend on the interface, loading at origin depends on the rotation $\theta_{0}=\partial_{, y} w_{0} ; \theta_{0}=\theta_{0}^{t}$. This rotation may be written [7] in appropriate form as $\theta_{0} \cong \frac{-a}{1+b}$ being:

$$
\begin{gathered}
a=\frac{\left(\alpha^{2}+\beta^{2}\right)}{o E^{\prime} \iota_{0}}\left[4 \alpha \beta e y_{0}+2 \beta z_{0}\right] \\
b=\frac{\left(\alpha^{2}+\beta^{2}\right)}{o E^{\prime} \iota_{0}}\left[-4 \alpha \beta e y_{0}+2 \beta z_{0}\right]
\end{gathered}
$$

\subsection{Profile Functions}

The numerical procedure presented above was coded into a FORTRAN routine and cases corresponding to some particular beams implemented. The procedure, though based in an implicit scheme, resulted in a code with fast rate of convergence. Results presented ahead, for the bending moment diagrams, come from use of this routine. The profile of the beam is set forth by the pair $\left\langle t, w_{i}\right\rangle$, where $t=t(y)$ is the function of thickness variation about the mean thickness $\bar{h}$ and $w_{i}=w_{i}(y)$ is the function describing the profile of the equal area axis. Harmonic forms are considered because it is supposed they are generated by the action of waves and wind, mostly. First function may be written as $t=1+\frac{a_{t}}{\bar{h}} \sin \left(\kappa_{t} y+\psi_{t}\right)$ being $a_{t}$ the 


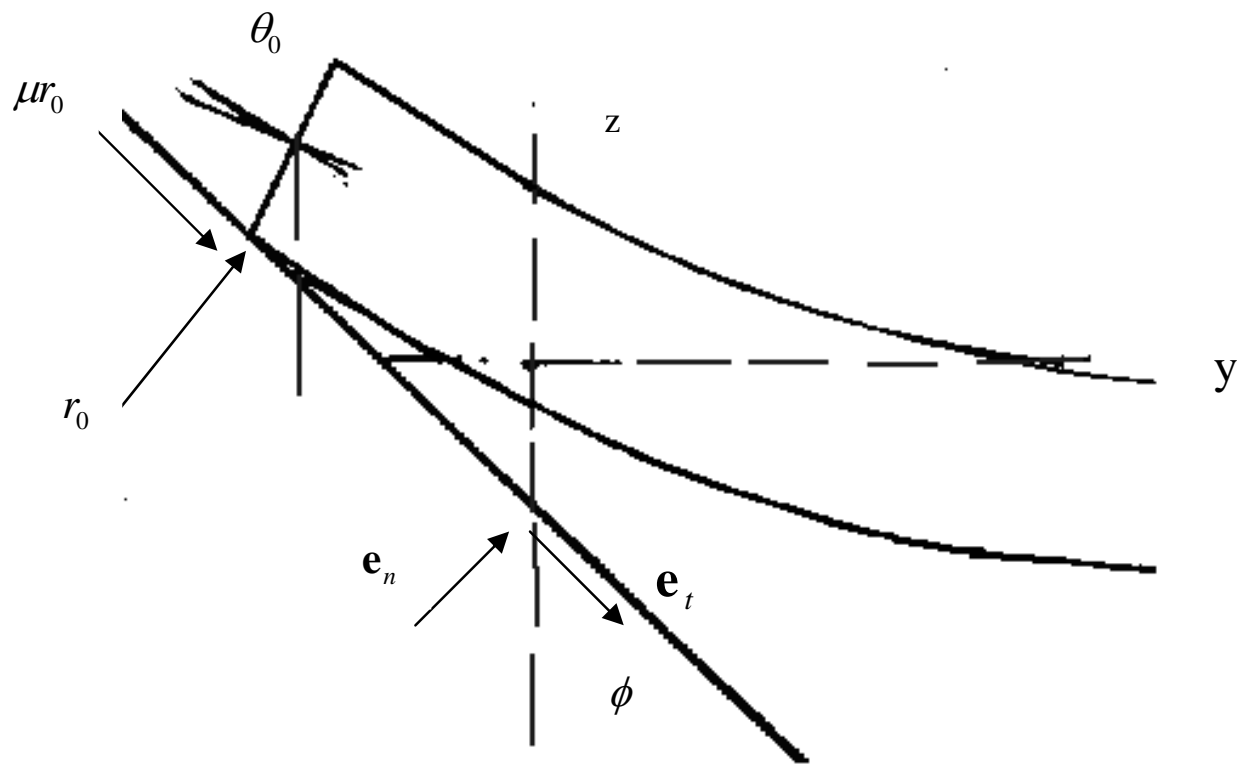

Fig. 3 Interface point loading scenario.

Table 1 Set of parameters used in the analysis.

\begin{tabular}{ll}
\hline Loading parameters & \\
\hline Slope angle set, degrees & $\phi=\{15,30,45\}$ \\
Eccentricity set & $\zeta=\{-0.5,0,0.5\}$ \\
Friction coefficient set & $\mu=\{0.05,0.25\}$ \\
\hline
\end{tabular}

Table 2 Properties of beam material.

\begin{tabular}{ll}
\hline Properties of ice & \\
\hline Elastic modulus $(\mathrm{Pa})$ & $E=0.50 e+10$ \\
Poisson's ratio & $v=0.30$ \\
Flexural strength (MPa) & $S_{f}=0.70$ \\
Compressive strength (MPa) & $S_{c}=5.0$ \\
\hline
\end{tabular}

amplitude, $k_{t}=\frac{2 \pi}{\lambda_{t}}$ the wave number, $\lambda_{t}$ the wave length and $\psi_{t}$ the phase angle. Table 3 presents the range of the used variables.

Second function $w_{i}$ is chosen to represent the profile of initial axis of the beam. It is related to the thickness profile. For harmonic-type imperfection profiles, non-symmetric case, a sinusoidal basis results:

$$
w_{i}=a_{i} \sin \left(\kappa_{i} y+\psi_{i}\right)
$$

where, $a_{i}$ is the amplitude of the sinusoidal, considered constant, $\kappa_{i}=2 \pi / \lambda_{i}$ is its wave-number-like parameter, being $\lambda_{i}$ the associated wave-length, and $\psi_{i}$ is the phase angle. The profile elements used are included in Table 4.

\section{Results}

\subsection{Thickness Variation Effect}

When $w_{i}=0$ in Eq. (1), only variations of thickness around the reference axis are present. Bending moment distribution in this case, fixed all parameters but the amplitudes, may be represented by a function $m=\hat{m}_{t / a_{t}}\left(y ; n_{0} ; a_{t}, \lambda_{t}, \psi_{t}\right)$. In Fig. 4 for $h_{0} / b_{0}=2$ and $\lambda_{t} / \lambda_{0}=0.125 ; \psi_{t}=0$, with boundary terms set to $\langle\phi=30, \mu=0.05, \zeta=0.50\rangle$, this is shown.

In it, moments are normalized with respect to the failure bending moment $m_{f}=\frac{2 i S_{f}}{h}$, being $S_{f}$ the flexural strength of ice. Positions along the beam are normalized with the wave-like factor $\lambda_{0}=\frac{2 \pi}{\delta_{0}}$. In-plane loads are normalized with the crushing load, $n_{c}=S_{c} h$. The plot shows a strong dependence of the 
Table 3 Thickness-variation-only function.

\begin{tabular}{ll}
\hline Parameters of thickness function \\
\hline Relative amplitudes & $\frac{a_{t}}{\bar{h}}=\{0,0.0625,0.125,0.1875\}$ \\
Relative length & $\frac{\lambda_{t}}{\lambda_{0}}=\{\infty, 0.500,0.125\}$ \\
Phase angle & $\psi_{t}=\{0,30,60,90\}$ \\
\hline
\end{tabular}

Table 4 Curvature-variation-only parameters.

\begin{tabular}{ll}
\hline Initial curvature function parameters \\
\hline Amplitude ratio & $\frac{\mathrm{a}_{\mathrm{i}}}{\mathrm{h}_{0}}=\{0,0.125,0.250\}$ \\
Wave-length ratio & $\frac{\lambda_{\mathrm{i}}}{\lambda_{0}}=\{0.125,0.250,0.500, \rightarrow \infty\}$ \\
Phase angle (deg) & $\psi_{i}=\{0,45,90,180,270\}$ \\
\hline
\end{tabular}

bending moments on the amplitude of the thickness variation function. In particular, it should be pointed out the closeness of the point of occurrence of the maximum bending moment with the position of minimum thickness.

Dependence of the bending moment field upon the wave length of the thickness profile is measured by $m=\hat{m}_{t / \lambda_{t}}\left(y, n_{0}, a_{t}, \lambda_{t}, \psi_{t}\right)$. These results are presented in Fig. 5 for $a_{t} / h_{0}=0.0625, \psi_{t}=0$ and the same boundary terms listed above. It can be observed there that negligible effects on bending moments occur at lengths of order $\lambda_{0}$-truly null effects only occur when $\lambda_{t} \rightarrow \infty$. Extreme values of bending moments occur at points close to minimum sectional thickness.

Additionally, because shorter wave lengths will bring the sections of minimum thickness closer to the loading region, they will be more effective in bringing up the extreme values of the bending moments.

A shift of phase angle $\psi_{t}$ increases the extreme values $m_{e}$ of bending moments when it brings the sections of minimum thickness closer to the loading region, as it is drawn in Fig. 6 for $m=\hat{m}_{t / \psi_{t}}\left(y, n_{0}, a_{t}, \lambda_{t}, \psi_{t}\right)$. In it boundary terms are conserved and $a_{t} / h_{0}=0.0625 ; \lambda_{t} / \lambda_{0}=0.125$. An opposite effect will be observed when it affects the first neck of the beam in an opposite sense.

\subsection{Initial Curvature Effect}

Similar to some extent is the behavior of the bending moment function as the effect of the initial curvature of the equal area axis only is considered. This response corresponds to the case where $t$ is set equal to $t_{0}$ in Eq. (1). Fig. 7 shows amplitude effect.

The other figures, Figs. 8 and 9, show the effect of wave length and phase angle of the initial curvature of the equal area axis on the bending moment behavior.

Overall, extreme values of bending moment $m_{e}=\hat{m}\left(y_{e} ; n_{0} ; a_{t}, \lambda_{t}, \psi_{t}\right)$, fixed the loading and geometry, occur at values $y_{e}^{m}$ such that $\partial_{, y}(m)_{y_{e}^{m}}=0$. Finding this position requires an internal loop added to the above procedure, as the value $y_{e}^{m}$ will not, in general, coincide with any discretized position $y_{n}$. Therefore, after an initial search that delimits this position, $y_{m} \leq y_{e}^{m} \leq y_{m+1}$ an approximate Newton procedure will find $\Delta y=y_{e}^{m}-y_{m}$ from $\Delta y=-\frac{\partial_{, y}(m)_{y_{m}}}{\partial_{, y y}(m)_{y_{m}}}$.

\subsection{Coupled Problem}

If thickness profile is unsymmetrical, lack of straightness of the equal area axis caused by sectional variations of thickness as well as thickness variations are present. These differ, however, from curvatures caused by interaction of ice features, due to inelastic effects mostly. They are geometric.

In such a case besides function $t$, function $w_{i}$ is required. At origin, value of $t=t(y)$ is $t_{0}$ while that of $w_{i}$ is $w_{\mathrm{i} 0}$. 

on Elastic Foundation under Contact Loading



Fig. 4 Effect of amplitude of thickness variation on bending moments.

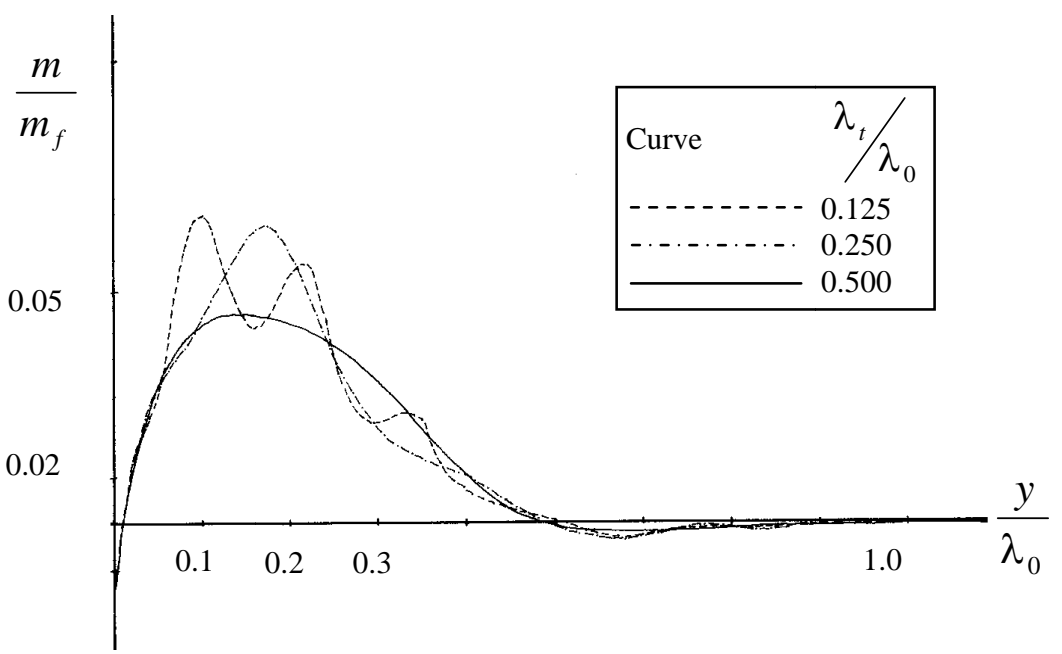

Fig. 5 Effect of wave-length of thickness variation on bending moments.

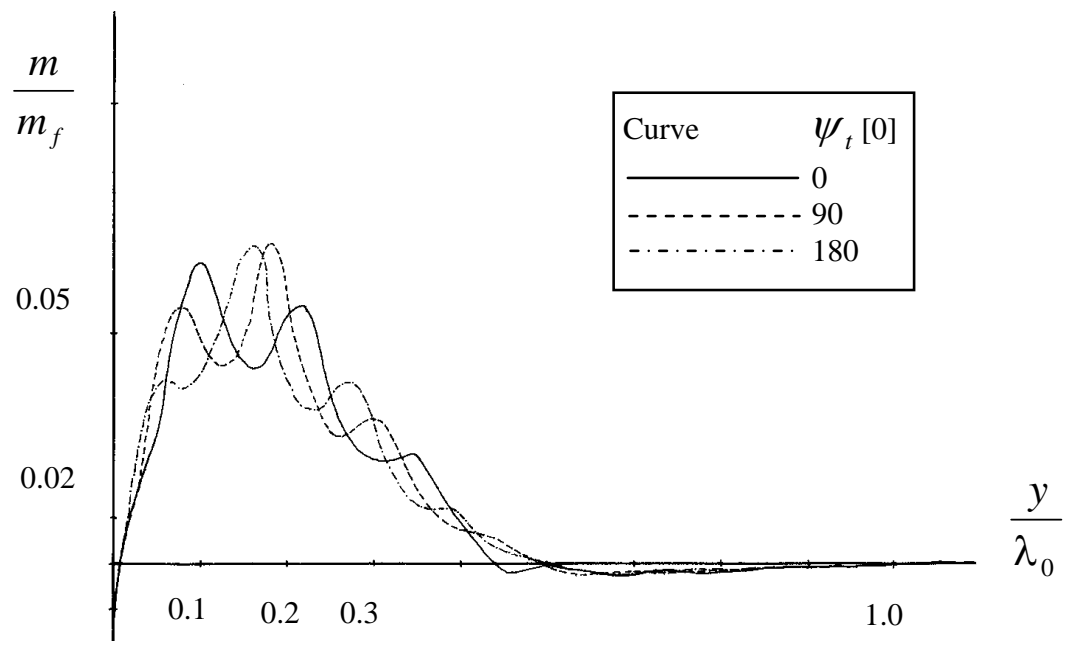

Fig. 6 Effect of phase angle of thickness variation on bending moments. 


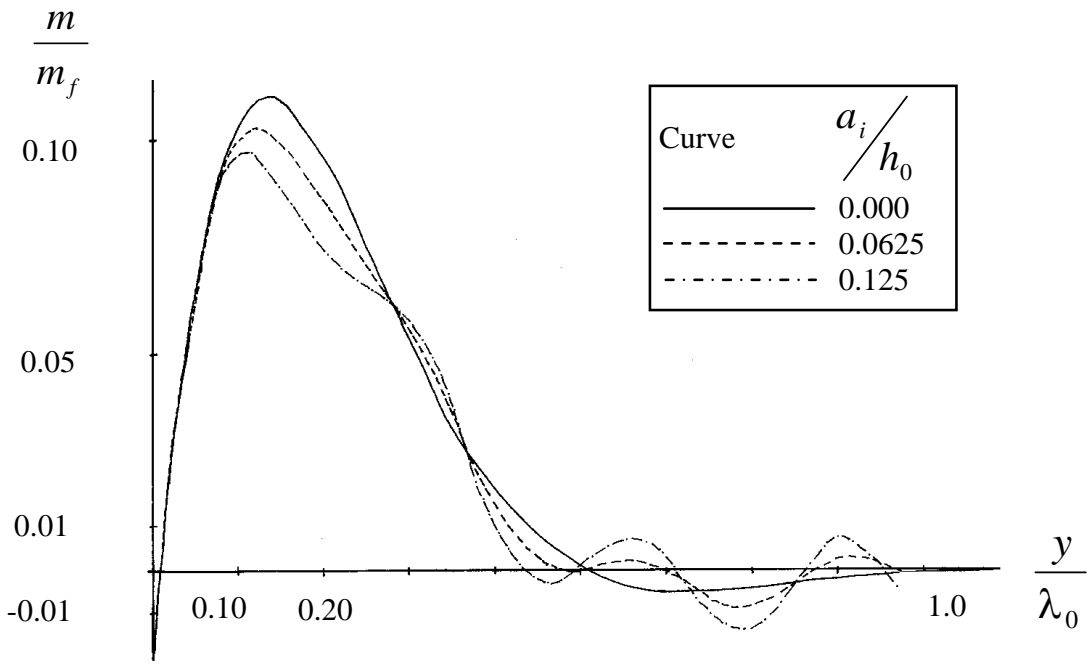

Fig. 7 Effect of amplitude of initial curvature on bending moments.

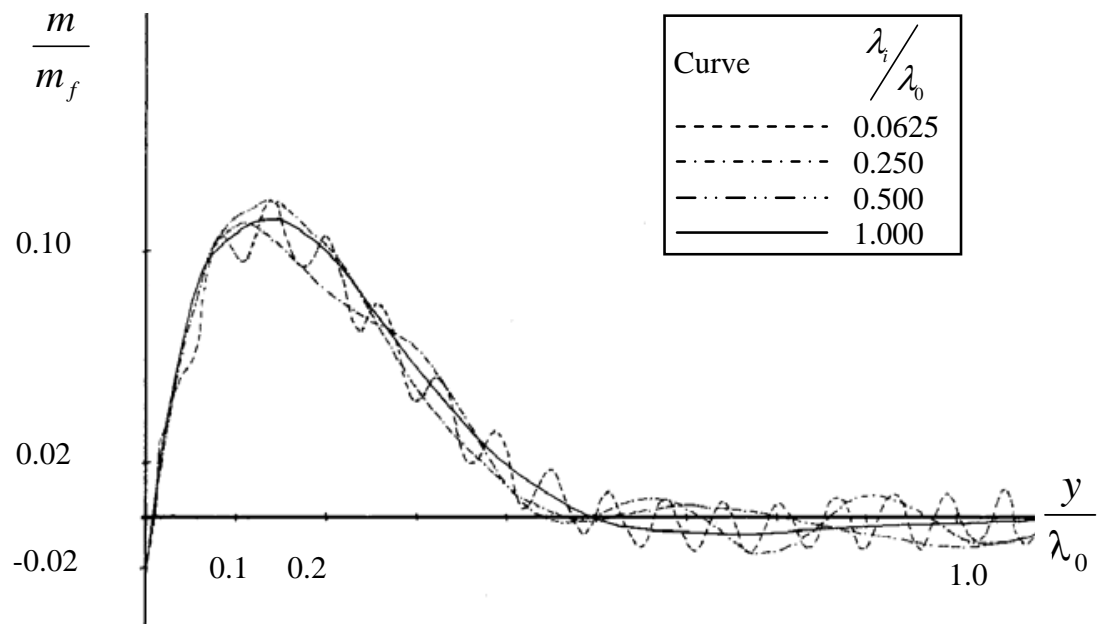

Fig. 8 Wave-length of initial curvature effect on bending moments.

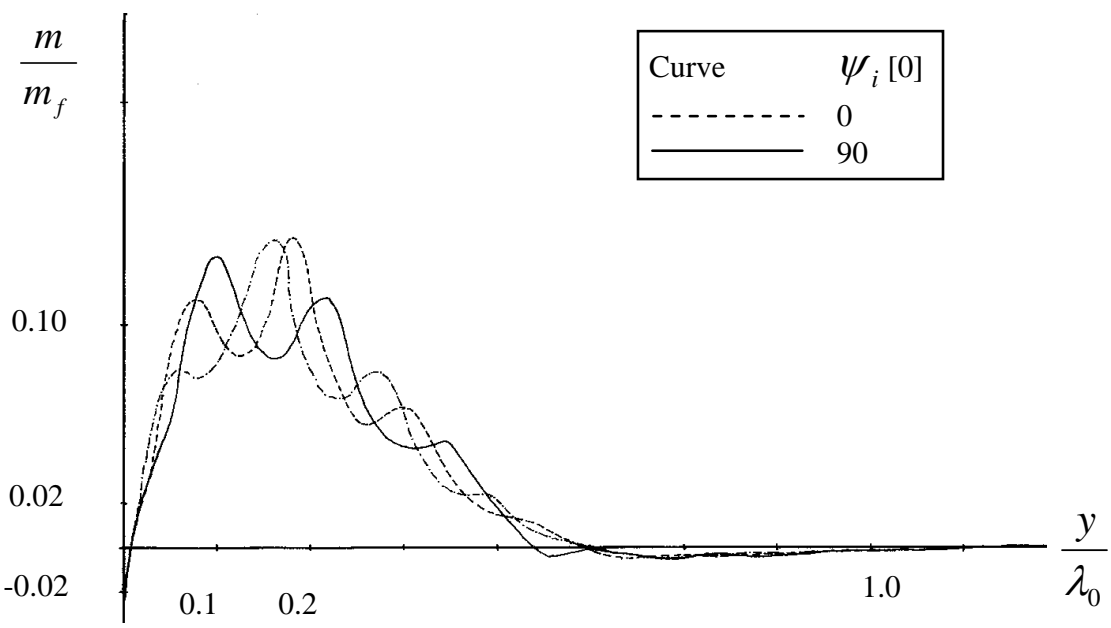

Fig. 9 Phase angle of initial curvature effect on bending moments. 


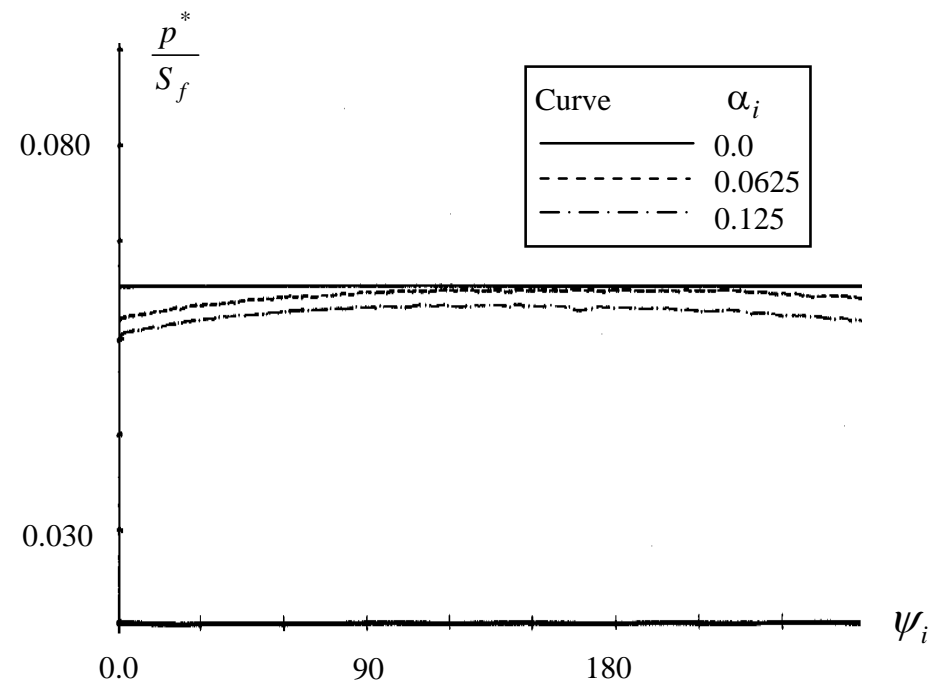

Fig. 10 Amplitude of imperfections effect on failure loads at thirty degree slopes.

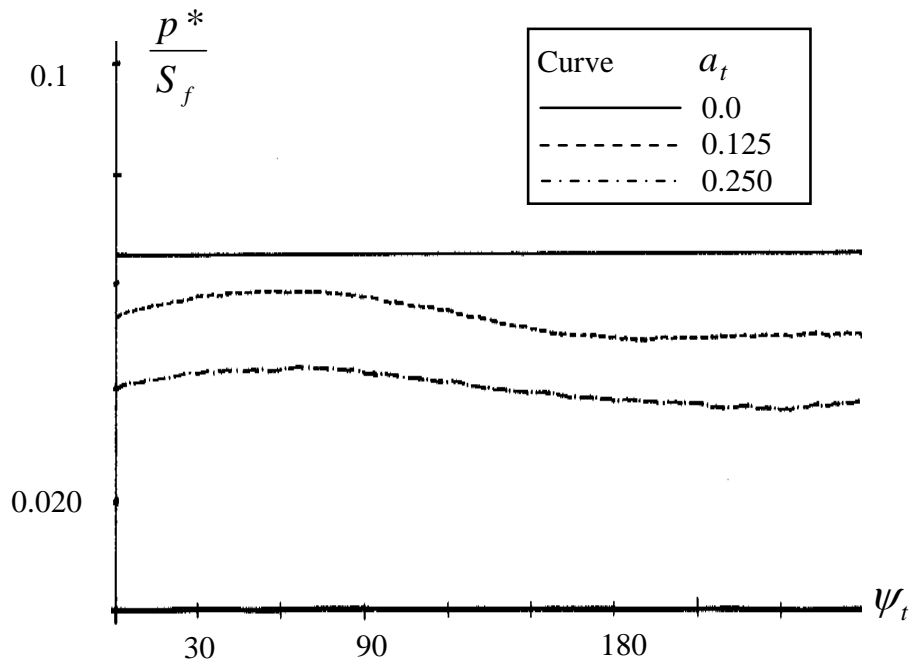

Fig. 11 Amplitude of thickness variation on failure loads at thirty degree slopes.

When curvature and thickness variations are present, the differential equation of equilibrium of beam element, Eq. (1) in its complete form has to be considered. Flexural displacements are referred to the initial curve of centroids, the equal area axis, but as in-plane loading is present, neutral axis does not coincide with this equal area axis. The distance $Z_{0}$ between them, however, is not large, in general, and may be disregarded, as in Eq. (1).

Solution of the coupled problem will lead to some modifications in the procedure presented above, but it still follows a parallel sequence to it.

\section{Failure Loads}

Failure loads may be characterized by a failure function $B^{t i}$ which defines the value of the maximum in-plane pressure, normalized with respect to the flexural strength the beam can withstand. Considering the traction side condition, extreme pressures $p^{*}=\frac{n_{0}^{*}}{b_{0} \bar{h} t_{0}}$ may be computed from the roots of the equation:

$$
\begin{aligned}
F=0, \quad F & =m-\frac{\bar{h} t_{o}}{6} n_{o}-\frac{\left(\bar{h} t_{o}\right)^{2}}{6} S_{f} ; \\
m & =m\left(y, n_{0} ; t, w_{i}\right)
\end{aligned}
$$


from the minimum value of normal $n_{0}{ }^{*}=\min \left(n_{0}\right) ; \quad \forall y$. This means then that:

$$
m_{, y}=0 ; \quad m_{, y}=m_{, y}\left(y_{e}, n_{0}^{*}\right)
$$

being $y_{e}$ the position of extreme value of bending moments. As Eq. (19) is implicit with respect to $n_{0}$, solution has to be obtained numerically using, for example, a Newton scheme. Once obtained, failure loads may then be represented as:

$$
B^{t i}=\frac{p^{*}}{S_{f}} ; \quad B^{t i}=\hat{B}^{t i}\left(\text { b.t. } ; h_{o} / b_{0}\right) ; w_{i} ; t
$$

This expression is dependent upon the boundary terms, b.t., aspect ratio, imperfection profile and thickness variation function. When the e.a.a. is straight and $t=1$, evidently $B^{t i}=B_{0} ; B^{0}=B^{0}\left(\right.$ b.t.,,$\left.h_{0}\right)$ being $B^{0}$ the failure function for perfect beams [8].

Contributions of functions $w_{i}$ and $t$ to the behavior of $B^{t i}$ may be considered individually. They are shown in the figures that follow, in the case of a beam with $h_{0} / b_{0}=2$. In the initial plot in Fig. 7, effect of amplitude $a_{i}$ of irregularity is shown for the case where $\lambda_{i} / \lambda_{0}=0.125$ with boundary terms $<\phi=30^{\circ} ; \mu=0.05 ; \zeta=0.50>$. For the same beam and same boundary terms, the thickness-variation-only effect is analyzed. In this figure, amplitude $a_{t}$ is considered when $\lambda_{t} / \lambda_{0}=0.125$ in Fig. 8.

For small deviations from the perfect beam case, in particular, a Taylor expansion may be used to approximate the function $B^{\mathrm{ti}}$ :

$$
B^{t i}=B^{0}+\left\lfloor\partial,_{\left\{w_{i}\right\}} B^{t i}\right\rfloor \cdot \Delta\left\{w_{i}\right\}+\left\lfloor\partial,_{\{t\}} B^{t i}\right\rfloor \Delta\{t\}
$$

where, the partial derivatives are computed at the evaluation position and $\left\{w_{i}\right\}$ is the column form of vector $w_{i}=\left\lfloor\begin{array}{lll}a_{i} & \kappa_{i} & \psi_{i}\end{array}\right\rfloor$ whereas $t=\left\lfloor\begin{array}{lll}a_{t} & \kappa_{t} & \psi_{t}\end{array}\right\rfloor$.

In Figs. 10 and 11, wavelength $\lambda_{i}$ and phase angles $\psi_{i}$ are considered. These factors present a mixed effect, increasing and decreasing the bending moments. In a softer manner, as compared to the amplitude effect, the plots show qualitatively.

\section{Conclusions}

Several conclusions may be drawn from the results derived from the implementation of the above model:

- Thickness variations by itself may be responsible for deviations of the order of $10 \%$, in some cases, in values of the failure pressures of ice beams;

- Initial curvature of the beam on its own may contribute to deviations of the same order of magnitude;

- Critical points in the thickness problem and initial curvature problem do not coincide, but are close, inclusive to the values based in uniform thickness, no initial curvature, beams. Sizes of broken blocks do not vary much;

- As the failure loads are directly related to the strength of ice, as temperature changes, these failure loads change accordingly;

- Variations of ice strength with temperature, and therefore across the beam height, should provide another important change in failure loads.

Use of these results still depends on mapping typical profiles of ice, and decomposition of them into harmonic terms, before implementing the above model. Clearly this may lead to a probabilistic approach of design. Alternatively, estimates of change of failure loads, computed with the present procedure, may be incorporated into design codes, based in uniform beam solutions. Moreover, the same idea may be extended to plate models, with variations of thickness, in order to build an enhanced understanding of the problem.

\section{References}

[1] S.F. Ackely, W.D. Hibler III, F. Kugzruk, A. Kovacs, W.F. Weeks, Thickness and Roughness Variation of Arctic Multi-year Sea Ice, CREEL report 78-13, New Hampshire, 1976.

[2] K.R. Croasdale, Ice Forces on Fixed, Rigid Structures, Cold Regions Research and Engineering Laboratory, 
Special report 80-26, Hanover, NH, 1980.

[3] J.B. de Aguiar, Bending failure of brittle plates and beams on an elastic foundation, Ph.D. Thesis, Massachusetts Institute of Technology, Massachusetts, 1987.

[4] K. Kubicek, M. Morek, Computational Methods in Bifurcation Theory and Dissipative Structures, Springer-Verlag, New York, 1983.

[5] G. Dahlquist, A. Bjorck, Numerical Methods in Scientific Computing, Society for Industrial and Applied Mathematics, Siam, Philadelphia, PA, 2008.

[6] M. Mellor, Mechanical Behavior of Sea Ice, Cold
Regions Research and Engineering Laboratory, Monograph 83-1, Hanover, NH, 1983.

[7] J.B. de Aguiar, 2D bending field of variable thickness floating ice beams loaded upon impact to an inclined wall, Latin American Journal of Solids and Structures 5 (2008) 10-15.

[8] J.B. De Aguiar, J.M. De Aguiar, On the deflections of semi-infinite irregular beams on an elastic foundation upon contact with a inclined wall, in: Anais IX Congresso Nacional de Engenharia Mecânica, CONEM 06, Recife, PE, Agosto, 2006. 